(С И.С. Гуль ${ }^{1}$, В.В. Осипов ${ }^{2}$, Д.Р. Ракита ${ }^{3}$, А.В. Соловьева ${ }^{4}$

Центр современной медицины «Доверие+», Рязань, Российская Федерация (1) ГБУ РО «Областная клиническая больница», Рязань, Российская Федерация (2)

Группа компаний «Развитие», Рязань, Российская Федерация (3)

Рязанский государственный медицинский университет имени академика И.П. Павлова, Рязань, Российская Федерация (4)

Цель. Изучить отдаленные результаты оперативного лечения гастроэзофагеальной рефлюксной болезни (ГЭРБ) в сочетании с хиатальной грыжей для оптимизации отбора пациентов для оперативного лечения.

Материалы и методы. Обследованы пациенты, прооперированные по поводу ГЭРБ в сочетании с хиатальной грыжей: 41 пациент в возрасте от 24 до 71 года. Пациентам были проведены следующие операции: лапароскопическая фундопликация по Toupet, лапароскопическая фундопликация по Nissen, неполная фундопликация по Черноусову. Одномоментно с лапароскопической фундопликацией ряду пациентов были проведены следующие вмешательства: крурорафия 36 больным, холецистэктомия по поводу желчнокаменной болезни - 10 пациентам и селективная проксимальная ваготомия 6 больным.

Результаты. Выявлено, что в послеоперационном периоде субъективные проявления ГЭРБ не всегда соответствуют степени ГЭРБ, определенной эндоскопически. Индекс массы тела - достоверный предиктор послеоперационного рецидива ГЭРБ и грыжи пищеводного отверстия диафрагмы, что необходимо учитывать при отборе пациентов к оперативному лечению.

Выводы. Операция лапароскопической фундопликации по Toupet с двусторонней крурорафией ассоциируется с лучшими послеоперационными результатами. Селективная проксимальная ваготомия в дополнение к антирефлюксной операции достоверно улучшает клинический результат.

Ключевые слова: гастроэзофагеальная рефлюксная болезнь, грыжа пищеводного отверстия диафрагмы, послеоперационный рецидив.

\title{
LONG-TERM RESULTS OF SURGICAL TREATMENT GASTROESOPHAGEAL REFLUX DISEASE IN COMBINATION HIATAL HERNIA
}

(C) I.S. Gul ${ }^{1}$, V.V. Osipov ${ }^{2}$, D.R. Rakita ${ }^{3}$, A.V. Solovieva ${ }^{4}$

Medical center «Doverie», Ryazan, Russian Federation (1)

Ryazan regional clinical hospital, Ryazan, Russian Federation (2)

Group of companies «Razvitie», Ryazan, Russian Federation (3)

Ryazan State Medical University, Ryazan, Russian Federation (4) 
Aim to study the long-term results of surgical treatment of gastroesophageal reflux disease (GERD) in combination with hiatal hernia to optimize the selection of patients for surgical treatment.

Materials and Methods. The study included 41 patients aged 24 to 71 years (mean age $49.9 \pm 4.95$ years), operated for gastroesophageal reflux disease in combination with hiatal hernia Patients underwent the following surgery: laparoscopic fundoplication according to Toupet, laparoscopic fundoplication according to Nissen, partial fundoplication according to Chernousov. The following interventions were performed simultaneously with laparoscopic fundoplication in a number of patients: cruroraphy in 36 patients, cholecystectomy for gallstone disease in 10 patients and selective proximal vagotomy in 6 patients.

Results. It is revealed that in the postoperative period subjective manifestations of GERD not always correspond to endoscopic degree of GERD. A body mass index is a reliable predictor of postoperative recurrence of GERD and hiatal hernia, that it is necessary to consider at selection of patients for surgical treatment.

Conclusions. Operation of a laparoscopic Toupet fundoplication with a bilateral krurorafiya is associated with the best postoperative results. The selective proximal vagotomy in addition to anti-reflux operation authentically improves clinical result.

Keywords: gastroesophageal reflux disease, hiatal hernia, postoperative recurrence.

Гастроэзофагеальная рефлюксная болезнь (ГЭРБ) представляет собой актуальную проблему не только для гастроэнтерологов, но и для всех специалистов терапевтического профиля. В результате эпидемиологического исследования ЦНИИ гастроэнтерологии частота выявления ГЭРБ составила 14,2\% [1]. Проведенное в городе Рязани в рамках многоцентрового исследования «Эпидемиология гастроэзофагеальной рефлюксной болезни в России» анкетирование выявило признаки ГЭРБ у $13 \%$ респондентов [2]. При сочетании ГЭРБ с грыжей пищеводного отверстия диафрагмы (ГПОД) пациент попадает в поле зрения хирургов, особенно при наличии показаний к оперативному лечению [3]. Наиболее часто отдаленные результаты оперативного лечения ГЭРБ и ГПОД анализируются хирургами с целью выбора оптимальной тактики лечения [4], с целью выявления факторов риска рецидива ГПОД, который по данным литературы составляет в среднем $25 \%$, достигая $42 \%$ при гигантских грыжах [5-7].

Несомненно, прооперированные пациенты нуждаются в динамическом наблюдении гастроэнтеролога для своевременной коррекции постфундопликацион- ного синдрома, частота которого составляет около $30-40 \%$ [8].

Цель исследования: изучить отдаленные результаты оперативного лечения гастроэзофагеальной рефлюксной болезни в сочетании с хиатальной грыжей для оптимизации отбора пациентов для оперативного лечения.

\section{Материалы и методы}

В хирургическом отделении ГБУ РО ОКБ по поводу ГЭРБ в сочетании с ГПОД прооперирован 41 пациент в возрасте от 24

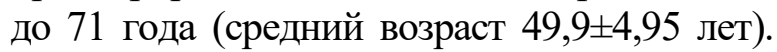
Из них женщины составили 75,6\% (31 человек), мужчины - 24,4\% (10 пациентов). Были проанализированы жалобы больных, анамнез, результаты объективного и дополнительного исследования, которое включало фиброгастроскопию (ФГС) и рентгеноскопию желудка с сульфатом бария. Информированное согласие на участие в исследовании было подписано всеми пациентами.

Статистический анализ полученных данных осуществляли при помощи пакета прикладных программ Statistica 6.0. Данные представлены в виде средней арифметической и ошибки средней арифметической $(\mathrm{M} \pm \mathrm{m})$. Корреляционный анализ проводили с определением коэффициента корреляции Спирмена (r). Критический уровень значи- 
мости (р) при проверке статистических гипотез принимали равным 0,05.

\section{Результаты и их обсуждение}

Пациентам, включенным в исследование, были проведены следующие операции (рис. 1): лапароскопическая фундопликация по Toupet (ЛФТ), лапароскопическая фундопликация по Nissen (ЛФН), неполная фундопликация по Черноусову (ФЧ). Од- номоментно с лапароскопической фундопликацией ряду пациентов были проведены следующие вмешательства: крурорафия 36 больным (ЛФТ2 - лапароскопическая фундопликация по Toupet с двусторонней крурорафией), холецистэктомия по поводу желчнокаменной болезни (ЖКБ) - 10 пациентам и селективная проксимальная ваготомия (СПВ) 6 больным.

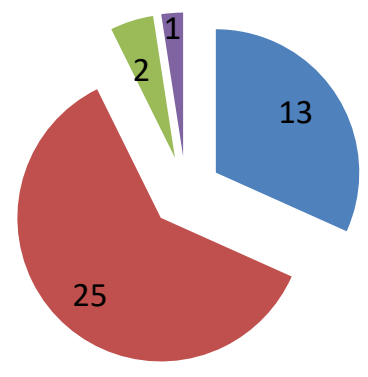

$$
\begin{aligned}
& \text { п ЛТ } \\
& \text { ЛФТ2 } \\
& \text { ЛФН } \\
& \text { ФЧ }
\end{aligned}
$$

Рис. 1. Оперативные вмешательства при ГЭРБ в сочетании с ГПОД

На момент обращения 8 пациентов (19,5\%), прооперированных по поводу ГЭРБ и ГПОД, не предъявляли жалоб. Жалобы остальных 33 пациентов представлены на рисунке № 2, наиболее частой жалобой явилась боль в эпигастрии $(45,4 \%)$ и отрыжка воздухом $(39,4 \%)$, изжога (27,3\%). Следует отметить, что корреляционный анализ продемонстрировал взаимосвязь между наличием жалоб в отдаленные сроки после оперативного лечения и величиной индекса массы тела (ИМТ) $(\mathrm{r}=0,3 ; \mathrm{p}=0,05)$. Интересным является факт отрицательной обратной связи субъективной изжоги у пациента и объективной степени эзофагита, определенной эндоскопически (r=-0,81; $\mathrm{p}=0,008)$.

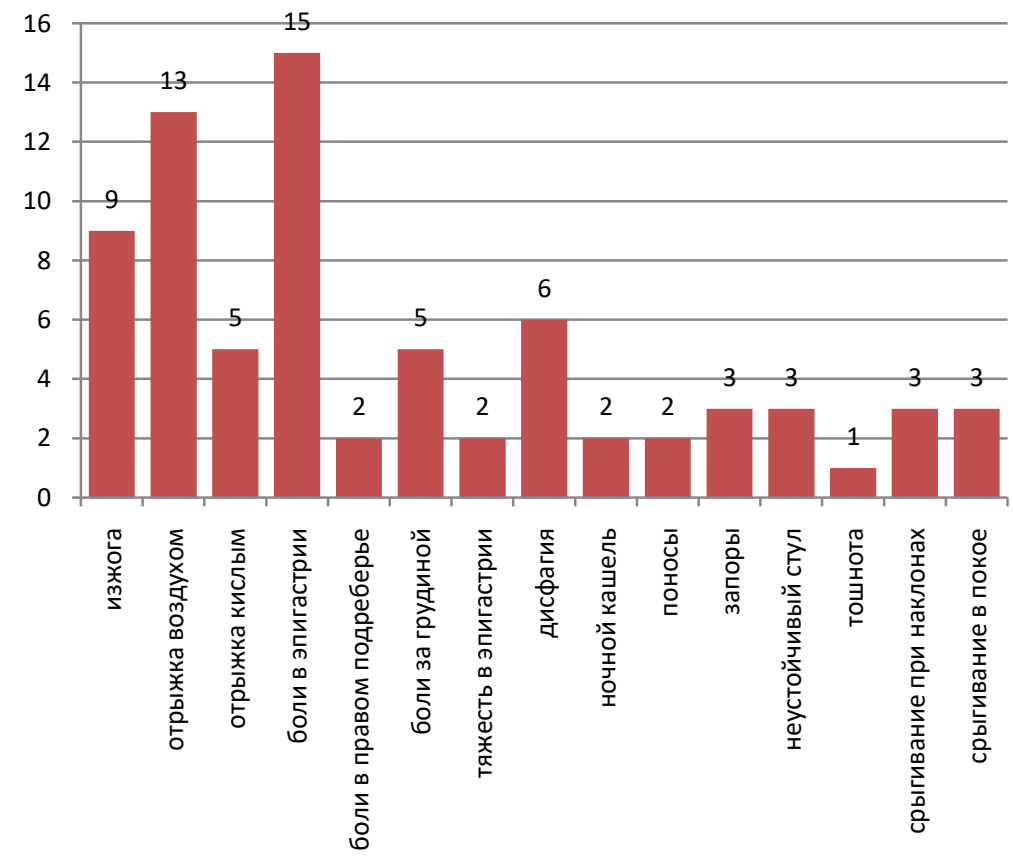

Рис. 2. Жалобы, предъявляемые пациентами в послеоперационном периоде 
Сопутствующая патология у обратившихся с жалобами пациентов представлена следующими нозологическими формами (рис. 3), среди которых преобладает абдоминальное ожирение в 70,7\% случаев и патология пищеварительной системы, представленная язвенной болезнью двенадцатиперстной кишки (ЯБ ДПК) (18\%), хроническим бескаменным холециститом (ХБХ) (24\%), постхолецистэктомическим синдромом (27\%).

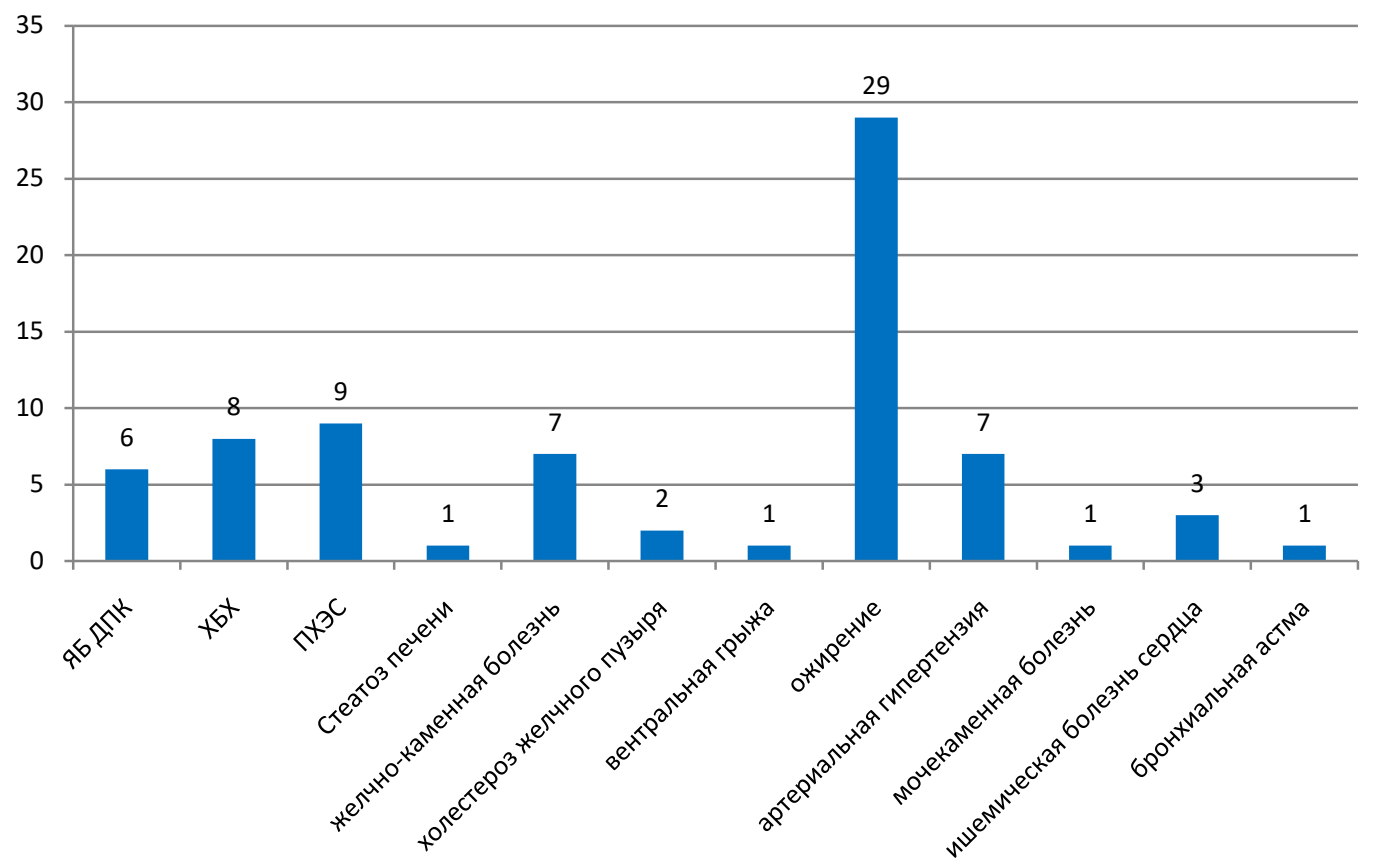

Рис. 3. Сопутствующая патология у больных, оперированных по поводу ГЭРБ и ГПОД

Важным, на наш взгляд, является анализ сроков обращения после операций, эти данные представлены на рисунке 4 . В сроки до 12 месяцев после операции обратилось 12 человек $(29,2 \%)$, так же как и через 4 года после оперативного лечения - ещё 12 паци- ентов. Что касается пациентов, обратившихся в сроки от 6 до 9 лет после операции (5 человек), то у них достоверно чаще диагностировалось укорочение пищевода $(\mathrm{r}=0,38 ; \mathrm{p}=0,01)$ и преобладающей жалобой была боль за грудиной $(\mathrm{r}=0,37 ; \mathrm{p}=0,014)$.

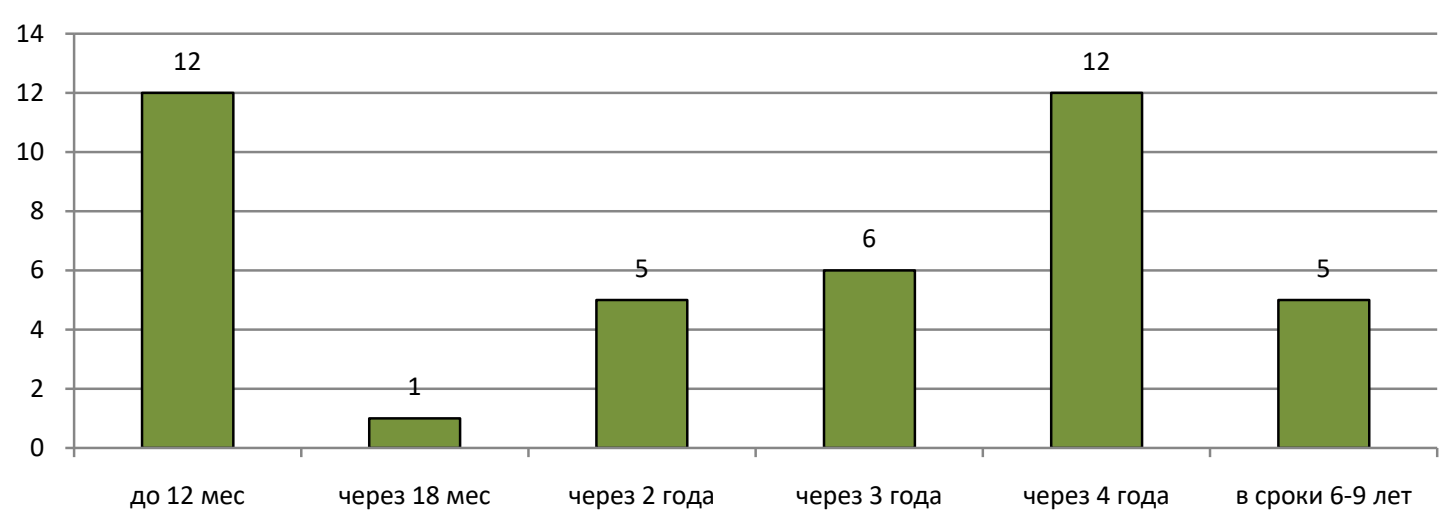

Рис. 4. Сроки обращаемости пациентов после операции 
При инструментальном обследовании пациентов выявлены следующие послеоперационные изменения - зияние кардии у 4 больных $(9,7 \%)$, неполное смыкание кардии - у 16 пациентов (39\%), гиперемия пищевода - у 20 (48,8\%), уменьшение расстояния от резцов до кардии зафиксировано у 9 больных $(21,9 \%)$. При проведении рентгеноскопии желудка с контрастированием ГПОД визуализирована у 22 пациентов $(53,6 \%)$, у 14 из них грыжа фиксированная; укорочение пищевода подтверждено у 9 больных (21,9\%).

Данные корреляционного анализа вновь указывают на роль абдоминального ожирения в послеоперационном рецидиве ГПОД: наличие ГПОД коррелировало с величиной ИМТ (r=0,44; $\mathrm{p}=0,003)$. У пациентов после лапароскопической фундопликации по Toupet с двусторонней крурорафией аксиальная кардиальная ГПОД встречалась достоверно реже ( $\mathrm{r}=-0,36$; $\mathrm{p}=0,02)$. Возвращаясь к объему антирефлюксных операций, необходимо отметить влияние СПВ на отсутствие жалоб в отдаленные сроки после оперативного лечения $(\mathrm{r}=0,31 ; \mathrm{p}=0,04)$.

\section{Выводы}

В послеоперационном периоде субъективные проявления гастроэзофагеальной рефлюксной болезни не всегда соответствуют степени рефлюкса, определенной эндоскопически. Индекс массы тела - достоверный предиктор послеоперационного рецидива гастроэзофагеальной рефлюксной болезни и грыжи пищеводного отверстия диафрагмы, что необходимо учитывать при отборе пациентов к оперативному лечению. Операция лапароскопической фундопликации по Toupet с двусторонней крурорафией ассоциируется с лучшими послеоперационными результатами. Селективная проксимальная ваготомия в дополнение к антирефлюксной операции достоверно улучшает клинический результат.

\section{Дополнительная информация}

Конфликт интересов: отсутствует.

Участие авторов:

Сбор данных, консультирование пациентов - И.С.Г., В.В.О.

Разработка концепции и дизайна исследования, анализ результатов - Д.Р.Р.

Статистическая обработка материала, анализ результатов, написание рукописи - А.В.С.
1. Лазебник Л.Б., Васильев Ю.В., Мананников И.В. Гастроэзофагеальная рефлюксная болезнь: эпидемиология, клинические аспекты, вопросы лечения // Справочник поликлинического врача. 2005. №3. С. 23-28.

2. Масленникова Е.В., Бутов М.А. Гастроэзофагеальная рефлюксная болезнь у жителей города Рязани (по данным эпидемиологического исследования) // Российский медико-биологический вестник имени академика И.П. Павлова. 2009. №1. С. 88-93. doi:10.17816/PAVLOVJ-20164112-118

3. Фалеев В.В., Федосеев А.В., Бутов М.А. Подход к лечению больных со скользящей грыжей пищеводного отверстия диафрагмы при наличии рефлюкс-эзофагита // Наука молодых (Eruditio Juvenium). 2017. №3. C. 435-446. doi:10.23888/HMJ20173435-446

4. Шарапов Т.Л., Бурмистров М.В., Сигал Е.И., и др. Профилактика осложнений при лапароскопических антирефлюксных операциях // Эндоскопическая хирургия. 2012. №3. С. 9-14.

5. Frantzides C.T., Madan A.K., Carlson M.A., et al. A prospective, randomized trial of laparoscopic polytetrafluoroethylene (PTFE) patch repair VS simple cruroplasty for large hiatal hernia // Arch. Surg. 2002. Vol. 137. P. 649-652.

6. Granderath F.A., Schweiger U.M., Kamolz T., et al. Laparoscopic Nissen fundoplication with prosthetic hiatal closure reduces postoperative intrathoracic wrap herniation: preliminary results of a prospective randomized functional and clinical study // Arch. Surg. 2005. Vol. 140, №1. P. 40-48.

7. Rathore M.A., Andrabi S.I., Bhatti M.I., et al. Meta-analysis of recurrence after laparoscopic repair of paraesophageal hernia // Journal of the Society of Laparoendoscopic Surgeons / Society of Laparoendoscopic Surgeons. 2007. Vol. 11, №4. P. 456-460.

8. Пучков К.В., Филимонов В.Б. Грыжи пищеводного отверстия диафрагмы. М.: Медпрактика; 2003.

\section{References}

1. Lazebnik LB, Vasil'ev YUV, Manannikov IV. Gastroehzofageal'naya reflyuksnaya bolezn': ehpidemiologiya, klinicheskie aspekty, voprosy lecheniya. Spravochnik poliklinicheskogo vracha. 2005; 3:23-8. (In Russ). 
2. Maslennikova EV, Butov MA. Gastroesophageal reflux disease at residents of ryazan (according to epidemiological research). I.P. Pavlov Russian Medical Biological Herald. 2009;17(1):88-93. (In Russ). doi:10.17816/PAVLOVJ20164112-118

3. Faleev VV, Fedoseev AV, Butov MA. Approach to treatment of patients with sliding hiatal hernia with underlying reflux esophagitis. Nauka molodykh (Eruditio Juvenium). 2017;5(3):435-46. doi:10.23888/HMJ20173435-446

4. Sharapov TL, Burmistrov MV, Sigal EI, et al. Prevention of complications of anti-reflux surgery. Endosk Hir. 2012;3:9-14. (In Russ).

5. Frantzides CT, Madan AK, Carlson MA, et al. A prospective, randomized trial of laparoscopic polytetrafluoroethylene (PTFE) patch repair VS simple cruroplasty for large hiatal hernia. Arch. Surg. 2002;137:649-52.

6. Granderath FA, Schweiger UM, Kamolz T, et al. Laparoscopic Nissen fundoplication with prosthetic hiatal closure reduces postoperative intrathoracic wrap herniation: preliminary results of a prospective randomized functional and clinical study. Arch Surg. 2005;140(1):40-8.

7. Rathore MA, Andrabi SI, Bhatti MI, et al. Metaanalysis of recurrence after laparoscopic repair of paraesophageal hernia. Journal of the Society of Laparoendoscopic Surgeons / Society of Laparoendoscopic Surgeons. 2007;11(4):456-60.

8. Puchkov KV, Filimonov VB. Gryzhi pishhevodnogo otverstiya diafragmy. Moscow: Medpraktika; 2003. (In Russ).

\section{Информация об авторах [Authors Info]}

* Соловьева Александра Викторовна - д.м.н., доцент кафедры факультетской терапии, Рязанский государственный медицинский университет имени академика И.П. Павлова, Рязань, Российская Федерация. e-mail: savva2005@bk.ru SPIN: 1943-7765, ORCID ID: 0000-0001-7896-6356, Researcher ID: U-9328-2018.

Alexandra V. Solovieva - MD, PhD, Assistant Professor of the Department of Faculty Therapy, Ryazan State Medical University, Ryazan, Russian Federation. e-mail: savva2005@bk.ru

SPIN: 1943-7765, ORCID ID: 0000-0001-7896-6356, Researcher ID: U-9328-2018.

Гуль Ирина Сергеевна - врач-гастроэнтеролог ЦСМ «Доверие+», Рязань.

Irina S. Gul - gastroenterologist of Medical Center «Doverie+», Ryazan, Russian Federation.

Осипов Владимир Владимирович - к.м.н., заведующий отделением лапароскопической хирургии №2, ГБУ РО Областная клиническая больница, Рязань, Российская Федерация.

Vladimir V. Osipov - PhD, Head of the Department of Laparoscopic Surgery №2, Regional Clinical Hospital, Ryazan, Russian Federation.

Ракита Дмитрий Романович - д.м.н., профессор, советник по медицинским вопросам, главный врач группы компаний «Развитие», Рязань, Российская Федерация.

SPIN: 4875-5719, ORCID ID: 0000-0001-9420-0962

Dmitry R. Rakita - MD, PhD, Professor, Medical Adviser, Chief Physician of the Group of Companies «Razvitie», Ryazan, Russian Federation. SPIN: 4875-5719, ORCID ID: 0000-0001-9420-0962.

Цитировать: Гуль И.С., Осипов В.В., Ракита Д.Р., Соловьева А.В. Отдаленные результаты оперативного лечения гастроэзофагеальной рефлюксной болезни в сочетании с грыжей пищеводного отверстия диафрагмы // Наука молодых (Eruditio Juvenium). 2018. T. 6, №4. C. 502-507. doi:10.23888/HMJ201864502-507

To cite this article: Gul IS, Osipov VV, Rakita DR, Solovieva AV. Long-term results of surgical treatment gastroesophageal reflux disease in combination hiatal hernia. Science of the young (Eruditio Juvenium). 2018;6(4):502-7. doi:10.23888/HMJ 201864502-507

Поступила / Received: 31.10.2018

Принята в печать / Accepted: 17.12 .2018 